\title{
Where Bell went wrong
}

\author{
Th. M. Nieuwenhuizen \\ Institute for Theoretical Physics, Valckenierstraat 65, 1018 XE Amsterdam, The Netherlands
}

\begin{abstract}
It is explained on a physical basis how contextuality allows Bell inequalities to be violated, without bringing an implication on locality or realism. Hereto we connect first to the local realistic theory Stochastic Electrodynamics, and then put the argument more broadly. Thus even if Bell Inequality Violation is demonstrated beyond reasonable doubt, it will have no say on local realism.
\end{abstract}

Quantum theory describes in my view the statistics of outcomes of measurements done on an underlying reality known to us as "Nature", while quantum mechanics or quantum field theory should be called "a theory", or "our present theory". In my view, in Nature particles are definite entities, subject to certain waves, that partly manifest themselves as the mysterious "quantum fluctuations". This view arose from studying the dynamics of quantum measurements ${ }^{1}$, and the subsequent question of what is going on in an individual quantum measurement. Some specific process must be going on in every individual measurement. We have no theory for that, but clearly Nature is using it, everyday the whole day. Quantum theory gives some admittedly strong, but incomplete information about outcomes of experiments.

One may also wonder what is going on in reality with cosmic rays, particles that have travelled to us for millions of years, or, even more stunning, with cosmic microwave background radiation, that travelled more than 13 billion years through empty space. These are not questions that should be answered within quantum mechanics, with answers like "In Hilbert space the state of a particle is represented by a state vector...", no, they are questions about what occurs in Nature. I see no other possibility than to assign a reality to cosmic ray particles and to photons, say "balls" or, preferably, "solitons", that travelled all these years to us through space.

"Quantum fluctuations" present a notion taught in any quantum mechanics course without anybody ever explaining what is fluctuating where. Quantum Mechanics and quantum field theory have the amazing property that we do not have to know these details if our aim is restricted to getting statistical predictions. This situation is somewhat reminiscent to the fact that, given a certain country, we don't have to speak its language to understand the statistics of its population such as the average age, average height, average income, and so on, of its people. But one cannot claim to understand the people without knowing their language. In other words: statistical understanding (quantum theory) is partial understanding that should never be taken for the full truth.

Stochastic Electromagnetism (SED) $)^{2}$ is to this date the most promising option to deal with the underlying level of reality. In that theory, "quantum fluctuations" are physical fluctuations of the classical electromagnetic field with a zero-point spectrum. Planck's constant enters by the strength of these fluctuations. A connection with quantum mechanics has been put forward by Cetto and de la Peña already some 15 years ago in their approach called "Linear Stochastic Electromagnetics", see e.g. ${ }^{2,3}$.

With quantum fluctuations expressed by SED or a comparable theory, and particles being solitons, the underlying quasi-deterministic level may be called "Stochastic Soliton Mechanics", a name I coined earlier in this series. ${ }^{4}$ Double slit interference should then emerge from solitons going through one of the slits and interfering with "idler waves" originating from the other slit.

In his opening address of the 2008 Växjö conference Foundations of Probability and Physics - 5, Andrei Khrennikov took the position that violations of Bell inequalities ${ }^{5}$ occur in Nature, but do not rule out local realism, due to contextuality: the measurements needed to test Bell inequalities (BI) such as the BCHSH inequality cannot be performed simultaneously ${ }^{6}$. Therefore Kolmogorian probability theory starts and ends with having different probability spaces, and Bell inequality violation (BIV) just proves that there cannot be a reduction to one common probability space. This finally implies that no conclusion can be drawn on local realism, since incompatible information can not be used to draw any conclusion. As explained below, the different pieces of the CHSH inequality involve fundamentally different distribution functions of the hidden variables, which cannot be put together in one over all covering distribution of all hidden variables of the set of considered experiments. To our knowledge, the first remarks related to contextuality were made by Cetto, Brody and de la Peña ${ }^{7}$. The contextuality position was first pointed at in mathematical rigor by Luigi Accardi ${ }^{8}$, and then taken by e.g. Fine ${ }^{9}$, Pitowsky ${ }^{10}$, Rastal ${ }^{11}$, Kupczynski ${ }^{12}$, Garola and Solombrino ${ }^{13}$ Khrennikov $^{14}$, Volovich ${ }^{15}$, Hess and Philipp ${ }^{16}$, Sozzo ${ }^{17}$ and Zhao, de Raedt and Michielsen ${ }^{18}$. Many of their contributions were reported in previous Växjö proceedings. I now also subscribe to this position.

At the University of Amsterdam I supervise bachelor projects on Bell inequalities. Students are happy to get insight in the possible structure of the physics behind the quantum formalism. The role of contextuality is a standard ingredient. Let us see how it comes up. 


\section{HOW THE CONTEXTUALITY ENTERS}

In the Clauser-Horne-Shimony-Holt (CHSH) setup, one may consider a source that emits pairs of spin $1 / 2$ particles, one going to station A "Alice" and the other, in opposite direction, to station B "Bob". At each station one out of two possible measurements is performed, $A_{1}$ or $A_{2}$ by Alice and $B_{1}$ or $B_{2}$ by Bob. In case $A_{1}$ the particle's spin is measured along the axis in direction $\mathbf{a}_{1}$ and in case $A_{2}$ along the axis in direction $\mathbf{a}_{2}$. Likewise $B_{1,2}$ corresponds to measurements along axis $\mathbf{b}_{1,2}$ respectively. The outcomes, "up" or "down" along axis $\mathbf{a}_{i}$ for A is denoted as $S_{A_{i}}= \pm 1$, respectively, and likewise for the measurement by B along axis $\mathbf{b}_{j}$ as $S_{B_{j}}= \pm 1$. The measurement is repeated many times. Ideally - if all particles of all pairs are measured - the recordings on the two detectors come as pairs. In each case, the direction of the axis is known and it is recorded whether "up" or "down" was measured. Putting afterwards the results from both detectors together, one determines the four correlators $C_{i j}=\left\langle S_{A_{i}} S_{B_{j}}\right\rangle$ for $i=1,2$ and $j=1,2$ by averaging the outcomes over the pairs. From these four objects one makes the combination proposed by Clauser, Horne, Shimony and Holt $(\mathrm{CHSH})^{19}$,

$$
B C H S H \equiv C_{11}+C_{12}-C_{21}+C_{22},
$$

where "B" stands for Bell. Since only \pm 1 variables are involved, it will clearly hold that each $\left|C_{i j}\right| \leq 1$ and $B C S H S \leq$ 4. But a stronger bound can be derived. Manipulating with ingredients inside the averages, one has

$$
B C H S H=\left\langle\left(S_{A_{1}}-S_{A_{2}}\right) S_{B_{1}}+\left(S_{A_{1}}+S_{A_{2}}\right) S_{B_{2}}\right\rangle .
$$

Because the $S$ variables are all \pm 1 , one of the two combinations will be zero, while the other is \pm 2 . This implies a version of the Bell inequalities,

$$
B C H S H \leq 2 .
$$

There are many papers that investigate in great rigor the validity of the steps made here, and they again lead to this result.

In the quantum mechanical description of the measurement, the state is supposed to be pure and described by the state vector $|\psi\rangle=\left(\left|\uparrow_{A}\right\rangle\left|\uparrow_{B}\right\rangle-\left|\downarrow_{A}\right\rangle\left|\downarrow_{B}\right\rangle\right) / \sqrt{2}$. The measurement of the particle's spin along axis $\mathbf{a}_{i}$ is described by the operator $\mathbf{a}_{i} \cdot \vec{\sigma}$, where $\vec{\sigma}=\left(\sigma_{x}, \sigma_{y}, \sigma_{z}\right)$ are the three Pauli matrices with $\sigma_{z}=\operatorname{diag}(1,-1)$ and we omit the prefactor $\frac{1}{2} \hbar$. Carrying out the manipulations, one finds that the maximum over the possible directions is taken when all vectors are in a plane, $\mathbf{a}_{1}$ is perpendicular to $\mathbf{a}_{2}$ and $\mathbf{b}_{1}$ perpendicular to $\mathbf{b}_{2}$, while the angle between $\mathbf{a}_{1}$ and $\mathbf{b}_{1}$ is $45^{\circ}$. The value is then $B C S H S=2 \sqrt{2} .^{19}$. In particular, a value $2<B C S H S<2 \sqrt{2}$ is allowed by quantum mechanics, but violates the Bell inequality (3).

We have not discussed how exactly the measurement is carried out, only that the results of all pairs were put together. It is standard to fix $i$ and $j$, say $i=1, j=2$ and then to collect enough measurement outcomes to allow a good statistical analysis. This is how one uses, say, a neutron beam, during, say, 30 minutes. Next, in a standard setup one changes either $i$ or $j$, and repeats the measurement during, say, another 30 minutes. In this way, the four correlators $C_{i j}$ are determined from consecutive measurements. This setup is good enough to get their values and to show that the Bell inequality (3) can be violated. It was applied in the first test of BIV by Freedman and Clauser ${ }^{20}$.

Bell, however, proposed to choose the measuring directions at A and B randomly from their two possibilities, at a moment well after the particles left the source, but well before they arrive at A and B. It is this selection procedure that brings in the issue of locality into the problem, that is to say, the question whether all speeds are less than the speed of light; if not, then the situation is called non-local. Now if the particles are separated from each other more than their travel time multiplied by the speed of light, and detector directions are randomly chosen, but happen to be in parallel directions, then it appears always that one of them gives an "up" registration and the other a "down" registration. From the point of angular momentum conservation this is obvious, but it is not obvious how the particles "get this done". Indeed, if the information about the - in this case parallel - directions of the measurement axes is know only when the particle distance is larger than ct, then, it seems, this information has to be transmitted between them with a speed larger than $c$. For this reason, Bell's conclusion is that BIV may point at non-locality. Alternatively, he noticed, it may be due to the fact that registered values are not related to properties the particles had before the measurement, a break of realism.

Either one or the other is broken, so BIV proves, according to Bell a breakdown of local realism. If this is true, it puts a major barrier to simple views on the reality underlying quantum theory and a rather hopeless starting point for attempts to improve on quantum theory by formulating a subquantum theory. Absence of local realism is counterintuitive, not to say awkward, so before giving it up, we should really have not any other option. 
Be it as it may, the first experiment along the lines devised by Bell was carried out by Aspect, Dalibard and Roger in 1982, thus consistent with having ruled out local realism ${ }^{21}$. Their work generated a whole field of research, with many contributions reported in Växjö meetings. So far, it is agreed that BIV occurs in many different systems, e.g. for photons, neutrons, ions and Kaons.

The contextuality issue arises in this discussion because in definition (1) we have put together correlators that could not be measured simultaneously. In particular, we have identified the averaging in the four terms, even though different things are averaged over. In the standard setup one measures them in separate runs. In the Bell-Aspect setup one randomly chooses the directions of the measurement axes, but by the time the particles arrive, it is set at some choice and from then on it looks as if it had been in that state all the time. So also then contextuality plays a role, and in the same way.

\section{HIDDEN VARIABLE MODELS}

\section{A. Bell's hidden variable description}

Bell considers that the measurements outcomes, the $S_{A_{i}}= \pm 1$ are determined by some set of hidden variables. Let us denote the set pertaining to the measured particles schematically by $\lambda$; being created as a pair, it is supposed that both particles both share the same set $\lambda$, that travels with them. It seems natural to follow Bell and assume

$$
C_{i j}=\int \mathrm{d} \lambda \rho(\lambda) S_{A_{i}}(\lambda) S_{B_{j}}(\lambda)
$$

In this way the four correlators all involve the same $\rho(\lambda)$. Therefore Eq. (3) can again be derived from (1) using (2), with angular brackets now denoting integrals over $\rho(\lambda)$. Since measurements hint that values $2<B C H S H<2 \sqrt{2}$ are possible, Bell concludes that local hidden variable models do not work and that Nature lacks local realism.

This argument seems so clear that most in the physics community are convinced that Bell is right.

\section{B. About loopholes}

Various loopholes are known. The first is the detection loophole - in experiments with photons at most $20 \%$ of them are detected. Such may lead to biases. It was closed in the experiment of Rohe et al. ${ }^{23}$. The second is the locality loophole: in experiments with ions the particles are not well separated, thus not excluding the possibility of information transfer at speeds lower than light. It was closed in the experiment of Weihs et al. ${ }^{24}$. For neutron double slit interferometry the spin information can not even in principal be separated from their path information ${ }^{25}$. Another case is the coincidence loophole: when can we speak about the detection of a pair ${ }^{26}$. Recently attention was payed to the fair sampling loophole. So far, fair sampling is a hidden assumption in the analysis of data, that cannot be checked. G. Adenier has defended in his PhD-thesis that BIV proves that the fair sampling assumption is violated, not local realism ${ }^{27}$.

Since after 25 years since the Aspect experiment it appears still to be very hard to close all loopholes in a single experiment it has been supposed, see e.g. $\operatorname{Santos}^{28}$ that Nature resists loophole-free Bell experiments. Still, this all is not our main theme. Our point will be that Bell went wrong even before the issue of these loopholes has to be addressed, because of the contextuality loophole, that cannot be closed.

\section{Improved hidden variables description}

Bell's argument would not convince Niels Bohr, since the detectors have not been taken into account. Clearly, the detectors consist of many particles and will also have hidden variables, $\lambda_{A_{i}}$ and $\lambda_{B_{j}}$. In this setup, each of the four correlators can be written as

$$
C_{i j}=\int \mathrm{d} \lambda \int \mathrm{d} \lambda_{A_{i}} \int \mathrm{d} \lambda_{B_{j}} \rho_{i j}\left(\lambda, \lambda_{A_{i}}, \lambda_{B_{j}}\right) S_{A_{i}}\left(\lambda, \lambda_{A_{i}}\right) S_{B_{j}}\left(\lambda, \lambda_{B_{j}}\right),
$$

where it is to be noted that we assume that the measured value at A does not involve any parameter of $\mathrm{B}$, hidden or not. To come back to the steps of Bell, one has to assume that the four $\rho_{i j}$ arise from one global distribution function $\rho_{G}$, so that, for instance, 


$$
\rho_{12}\left(\lambda, \lambda_{A_{1}}, \lambda_{B_{2}}\right)=\int \mathrm{d} \lambda_{A_{2}} \int \mathrm{d} \lambda_{B_{1}} \rho_{G}\left(\lambda, \lambda_{A_{1}}, \lambda_{A_{2}}, \lambda_{B_{1}}, \lambda_{B_{2}}\right) .
$$

and likewise for $\rho_{11}, \rho_{21}, \rho_{22}$. This is the new way in which contextuality enters: it is assumed that there exists an underlying distribution $\rho_{G}$ of the sets of hidden variables of all the measurements, even though they cannot be carried out simultaneously.

With this relation, the manipulations that led from (1) to (3) can be repeated and the same Bell inequality can be derived. It being violated in experiment, one then concludes that Bell was basically right, the detectors bring no new information, and hidden variable models are excluded. The remaining focus is then to close the loopholes and prove that Bell was indeed right.

\section{QUANTUM MEASUREMENTS}

One point overlooked by Bell is thus the role of the hidden variables of the detectors. Contrary to Bohr's philosophy, Bell did not include them explicitly in his considerations. This may be blamed to an attitude created by the projection postulate of quantum mechanics. In textbooks it is mostly postulated that a quantum measurement amounts to a non-unitary projection of the quantum state on an eigenstate of the measured operator. This idealized notion is completely different from what is common practice in experimental laboratories. Indeed, quantum measurements are dynamical processes too that can be described within quantum mechanics.

Indeed, it has been possible to consider a rich enough mode ${ }^{1}$ for an apparatus that can perform the measurement of a spin $\frac{1}{2}$. The apparatus is an Ising magnet, consisting of a large number of quantum spins $\frac{1}{2}$, coupled to each other only via their z-components (Ising character) and also coupled to a bath. The magnet starts out in a metastable paramagnetic state, and by coupling to the tested spin, the magnetization is driven into its stable up- or down ferromagnetic state. Here the metastability offers a multiplication of the weak quantum signal of the tested spin into the macroscopic, stable up- or down value of the magnetization at the end of the measurement, that is easily recorded. The bath is also needed, namely for dumping the excess (free) energy from the initial state of high (free) energy. In this model, the Schrödinger cat states disappear quickly, first by an NMR-type dephasing due to the interaction of the tested spin with the spins of the magnet, and then, in the dephased situation, all memory of the initial state is erased by decoherence due to the coupling to the bath ${ }^{1}$.

This approach thus describes a quantum measurement as a specific process of quantum mechanics, in which two important timescales are concerned: the small dephasing time and the somewhat larger decoherence time of the offdiagonal terms, and the larger registration time of the diagonal elements, that is, the time in which the up- or down magnetization is built up. If all are still rather small, one may consider these processes effectively as "instantaneous" and the collapse as a non-unitary evolution. This is what is taught in most textbooks, and we stress that to an extent it is misleading. The collapse view holds only in an effective sense, in reality the complete dynamics is unitary in the full Hilbert space of tested system and the apparatus. From the point of view of the tested system, it is an open system dynamics.

\section{A. Where did Bell make the error?}

The error of Bell lies in the assumption of the existence of a $\rho_{G}$, see Eq. (6). This was not explicit, since Bell did not include the role of detectors in his formulas. It was an implicit error, due to the hidden assumption that detectors could be left out in the final argument.

But it is absolutely not true that, if one knows the marginals, here the $\rho_{i j}\left(\lambda, \lambda_{A_{i}}, \lambda_{B_{j}}\right)$ for $i=1,2$ and $j=1,2$, one may conclude that a common distribution $\rho_{G}$ exists. There are theorems on this and there are explicit examples in which some probabilities then have to be negative ${ }^{22}$. The latter does not make sense, so it is better to say that a common $\rho_{G}$ does not exist. Physically this is not a complete surprise, because anyhow the relevant experiments could not be carried out simultaneously. This uncomfortable knowledge thus appears to express itself also by absence of a common probability distribution (mathematicians say: absence of a common probability space).

\section{STOCHASTIC ELECTRODYNAMICS}

So far, so good, the above is common knowledge - even though not commonly accepted. On my way back from the Växjö 2008 conference Foundations of Probability and Physics-5 to my hometown Amsterdam, I realized at the 
airport of Copenhagen - it had to be there - that a physical argument can be brought into the discussion of hidden variable theories such as SED and alikes. In such theories there are specific hidden variables, those that, at some initial time, set the stochastic forces acting on the measurement apparatuses. A different setting of an apparatus corresponds to a physically different situation and thus to physically different sets of these hidden variables. In each setting, they drive the quantum working of the relevant apparatus, including opposite outcomes when members of a pair are measured along parallel axes. There is no physical reason why for different apparatuses the hidden variables should have the same nature, that is, have a common distribution, that is, be defined on a common probability space.

This can be made more explicit by imagining that when Alice's detector is in direction $\mathbf{a}_{1}$, there will be put some other apparatus in direction $\mathbf{a}_{2}$. It is immaterial what this is exactly doing, but for sure it will be driven by the hidden variables that would drive Alice's detector were it in this direction. Now it is clear that we speak about physically exclusive situations, each setup $A_{1,2}$ is distinctive and it excludes the other, $A_{2,1}$ : One can't have the cake and eat it. Again, for this very reason there is no justification to assume that their hidden variables are described by a common distribution $\rho_{G}$.

In any hidden variable theory, one may expect emission of radiation by Lorentz damping, i. e. accelerated electrons. This is a physical effect, which in SED is statistically balanced by the stochastic forces to reach an equilibrium "quantum" state, due to the presence of a fluctuation-dissipation theorem ${ }^{2}$. This brings once more a physical aspect of detectors, once more precluding attempts to put different setups together. Even if this Lorentz damping is not measurable nowadays, we only need to think of the heating of air by the apparatus (and by the second, irrelevant one, if it is there), surely a measurable effect, to realize that different setups of the detectors exclude each other and thus have no cause for possessing a common hidden variables distribution.

Within SED there is a clear understanding of the Bell-Aspect switching of detector directions: this just has no influence. What counts is the position of the detector at the moment when the particle arrives, not what happens before. Freedman and Clauser already employed this fact when considering detectors without random switching ${ }^{20}$.

David Mermin has formulated a pedagogic model where the members of the particle pairs carry instruction sets for the outcomes of the detectors, which contradicts this conclusion ${ }^{29}$. However, Adenier showed that his model can reproduce quantum results if non-detection events are included in the instruction sets ${ }^{30}$.

\section{CONCLUSION}

Violations of Bell inequalities occur in Nature if loophole-free situations can be reached. The BIV of quantum physics are adequately explained by quantum theory. Though loopholes have yet not been closed, we would be very surprised if quantum theory would not give the right answer. Indeed, if it did not, then how could it work so well otherwise?

It has always stunned me that Bell's simple hidden variables argument could have such profound implications as the absence of local reality. With some experience in deriving physical results to explain observations in various fields, the Bell analysis has always appeared rather abstract (mathematical) and suspiciously simple to me. The above concrete step of a physical implementation of the contextuality argument makes clear to me on a physical basis that Bell just overlooked a mathematical issue. The contextuality argument puts his conclusion where it should be: a mathematical derivation devoid of a clear physical mechanism, that can be refuted on the basis of proper mathematics, contextuality, or, as we showed, on the basis of physics, exclusiveness of different detector setups.

So far, in literature it is claimed only that a violation of Bell inequalities leads to absence of a common distribution. Our physical argument makes clear that it must also be absent when the BI are not violated.

Assuming a common distribution function for hidden variables of incompatible experimental setups is like adding apples to oranges. It is know that two apples plus three oranges do not add up to five bananas. Likewise, even when combining the outcomes of results of incompatible setups does lead to results described by quantum theory, this managing of data does not yield information about deep physical properties such as locality or realism. The physical input is much too poor to address those physical questions. They are, in my view, out of sight of the progress in physics that we may hope for in next decades.

Bell inequalities are of profound physical interest, as ever, but they have no say on local reality. Experimental tests of non-local realism, though reported in Ref. ${ }^{31}$ in connection with Bell inequalities, are actually far beyond the present level of understanding and manipulation. Nature may possess local realism or not, Bell inequalities have no say on that. For now we can just keep our cards on the familiar assumption of Nature possessing local realism.

As for searching the local reality underlying quantum theory, I conclude that Bell has kept us nicely busy, by obscuring the goal. We shall gratefully forgive him, he asked important questions and his efforts led to new fields such as quantum communication and quantum cryptography. But abstract mathematical reasoning has a faint chance to capture relevant physical mechanisms, and once again this was the lesson to learn. Now it is time to get physics back 
to the forum of particles, forces and hidden variables. It is really time to move on!

1 A. E. Allahverdyan, R. Balian and Th. M. Nieuwenhuizen, Europhys. Lett. 61, 452 (2003).

${ }^{2}$ L. de la Peña and A. M. Cetto, The Quantum Dice: An Introduction to Stochastic Electrodynamics, (Kluwer, Dordrecht, 1996).

${ }^{3}$ L. de la Peña and A. M. Cetto, in Quantum Theory: Reconsideration of Foundations - 3, G. Adenier, A. Yu. Khrennikov and Th.M. Nieuwenhuizen, eds, (AIP Conference Proceedings 810, Melville, 2006), pp 131.

${ }^{4}$ Th. M. Nieuwenhuizen, AIP Conf. Proc. 810, Quantum Theory: Reconsideration of Foundations - 3, G. Adenier, A. Yu. Khrennikov and Th. M. Nieuwenhuizen, eds. (Am. Inst. Phys., Melville, NY, 2006), pp 198.

5 J. S. Bell, Speakable and unspeakable in quantum mechanics, (Cambridge Univ. Press, Cambridge, 1987).

${ }^{6}$ A. Yu. Khrennikov, arXiv:0709.3909.

7 A. M. Cetto, T. Brody and L. de la Peña, Lett. Nuovo Cim. 5, 177 (197).

${ }^{8}$ L. Accardi, Phys. Rep. 77 (1981) 169.

9 A. Fine, Phys. Rev. Lett. 48, 291295 (1982).

10 I. Pitowsky, Phys. Rev. Lett. 48, 1299-1302 (1982).

${ }^{11}$ P. Rastal, Found. of Physics 13, 555-575 (1983).

12 M. Kupczynski, Phys. Lett A 116, 417-422 (1986).

${ }_{13}$ C. Garola and L. Solombrino, Found. Phys. 26, 1121 (1996).

14 A. Yu. Khrennikov, Found. of Phys. 32, 1159-1174 (2002).

15 I. V. Volovich, in Proc. Conf. Quantum Theory: Reconsideration of Foundations, ed. A. Yu. Khrennikov, Ser. Math. Modeling, Växjö Univ. Press, Växj”o 2, pp. 423 (2002).

${ }^{16}$ K. Hess and W. Philipp, in Proc. Conf. Foundations of Probability and Physics-3, Edited A. Yu. Khrennikov, AIP Conference Proceedings, American Institute of Physics, New York, Vol. 750, pp. 150-155 (2005).

17 C. Garola and S. Sozzo, quant-ph/0703260 (2007).

18 S. Zhao, H. de Raedt and K. Michielsen, Found. Phys. 38, 322 (2008).

19 J. F. Clauser, M.A. Horne, A. Shimony, R. A. Holt, Phys. Rev. Letters 49, 1804 (1969).

20 S. J. Freedman and J. F. Clauser, Phys. Rev. Lett. 28, 938 (1972).

${ }^{21}$ A. Aspect, J. Dalibard, G. Roger, Phys. Rev. Lett. 49, 1804 (1982).

22 N. N. Vorobev, Theory of Probability and its Applications 7, 147-162 (1962).

${ }_{23}$ M. A. Rohe, D. Kielpinski, V. Meyer, C. A. Sackett, W. M. Itano, C. Monroe and D. J. Wineland, Nature 409, 791 (2001).

${ }^{24}$ G. Weihs, T. Jennewein, C. Simon, H. Weinfurther and A. Zeilinger, Phys. Rev. Lett. 81, 5039 (1998).

25 Y. Hasegaga, R. Loidl, G. Barudek, M. Baron and H. Rauch, Nature 425, 45 (2003).

${ }^{26}$ J. A. Larsson and R. D. Gill, Europhys. Lett. 67, 707 (2004).

27 G. Adenier, Local realist Approach and Numerical Simulations of Nonclassical Experiments in Quantum Mechanics, Thesis, Växjö University, November 2008.

${ }^{28}$ E. Santos, arXiv:quant-ph/0410193 (2004).

${ }^{29}$ N. D. Mermin, Am. J. Phys. 49, 940 (1981).

${ }^{30}$ G. Adenier, Am. J. Phys. 76, 147 (2008).

${ }^{31}$ S. Gröblacher, T. Paterek, R. Kaltenbaek, C. Brukner, M. Zukowski, M. Aspelmeyer and A. Zeilinger, An experimental test of non-local realism, Nature 446, 871 (2007). 